\title{
Interacción social y nivel de conflicto sociocognoscitivo en la construcción del esquema de probabilidad
}

\author{
Pablo Fernández Berrocal \\ Universidad de Málaga
}

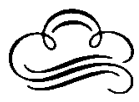

\section{Resumen}

En este estudio se analiza la influencia que la interacción social y el conflicto sociocognitivo tienen en la construcción del esquema de probabilidad por medio de un diseño pretest-intervención-postest.

Se muestra cómo las situaciones experimentales de interacción social con conflicto sociocognitivo producen mayores logros durante la fase de intervención, asi como mayores avances cognitivos en el postest en el esquema de probabilidad que las condiciones de trabajo individual y las de interacción sin conflicto. Los progresos alcanzados en el postest para el esquema de probabilidad se generalizaron al esquema de proporciones.

\section{Abstract}

The purpose of this work is to analyze the social interaction and socio-cognitive conflict influence on the construction of the probability scheme by on pretest-intervention-postest design.

It could be showed bow social interaction with socio-cognitive conflict in experimental situations produce bigher outcomes during the intervention fase as well as more cognitive advances on the probability schemes than on the individual work conditions and social interaction without socio-cognitive conflict during the postest fase. Achieved outcomes on the probability scheme were generaliced to the proportions scheme.

Agradecimientos: Desearía agradecer a Alfredo Fierro Bardají la supervisión y seguimiento de esta investigación, así como la lectura y discusión de este manuscrito. También la colaboración y apoyo intelectual de Gerardo Echeita.

Dirección del autor: Pablo Fernández Berrocal, Dpto. Psicología Básica, Metodología y Psicobiología, Campus Teatinos. 29071 MALAGA 
... la ciencia y la moral se realizan a partir de los acuerdos $y$ desacuerdos entre los bombres en interacción intima. (Cattaneo, 1864).

\section{INTRODUCCION}

Esta novedosa idea de Carlo Cattaneo sobre la adquisición y desarrollo del conocimiento fue arrinconada por la historia. Autores posteriores como Baldwin (1913), Mead (1934), Wallon (1947), Vygotski (1934) propusieron formulaciones más explícitas y sistematizadas de la influencia de lo social en lo cognitivo, aunque no ha sido hasta muy recientemente que se ha tomado con cierta seriedad dichas propuestas (Doise y Mugny, 1981; Wertsch, 1979; 1985).

Con seriedad, pero tambioén con timidez, puesto que en la Psicología del Pensamiento predominan, aún, enfoques como el piagetiano sobre la adquisición del conocimiento que priman los factores estructurales y lógicos sobre los sociales, como ha señalado Rivière, "Piaget, sí, habla del conocimiento como producto de una interacción, pero de hecho restringe fundamentalmente esa interacción a la relación entre el sujeto y los objetos físicos, y enraiza las formas de la razón, las estructuras operatorias, en la acción sobre el mundo físico, y no en la interrelación con el mundo social.» (Rivière, 1987; pág. 86).

De forma similar, los actuales desarrollos desde el procesamiento de la información (Anderson, 1980; Fodor, 1983) han persistido en aislar al «sujeto cognitivo» de su contexto sociocultural y relacional (Bruner, 1986; De Vega, 1985; Echeita, 1988; Edwards y Mercer, 1987; Lave, 1988; Rivière, 1987; Seoane, 1985).

En el campo del razonamiento los primeros estudios que se han atrevido intelectualmente a enfrentarse con dicho reto han sido los de Forman y Cazden (1985) y los del grupo ginebrino Doise y Mugny (1981) y Perret-Clermont (1979), que han intentado plasmar y profundizar con sus investigaciones en el postulado vygotskiano:

Todas las funciones superiores se originan como relaciones entre seres humanos. (Vygotski, 1978; pág. 94).

Aunque aducirán mecanismos diferentes para este proceso. Los primeros, los más cercanos a las tesis de Vygotski, plantean que los logros cognitivos se alcanzan por la coordinación de sus habilidades particulares para construir de forma conjunta una solución a un problema; en cambio, los segundos, con los pies todavía en Piaget, señalan que la función de la interacción social es la de generar «perturbaciones» o conflictos sociocognitivos en el sistema de creencias de los sujetos. Profundizaremos en este último enfoque porque es el que ha incitado la investigación que presentamos.

Además de Piaget $(1923,1924,1932)$, el conflicto como suscitador de progresos cognitivos había sido sugerido y desarrollado por diversos autores (Murray, 1972; Berlyne, 1957; Kuhn, 1972; Smith, Johnson y Johnson, 1981), pero se trataba de un conflicto puramente cognitivo. Este nuevo enfoque pretende estudiar el conflicto desde un punto de vista social y no únicamente desde un punto de vista cognitivo. Esta dimensión social del conflicto que ha estado escindida de la investigación es tomada como «leitmotiv» de los trabajos del mencionado grupo ginebrino.

El conflicto sociocognitivo, entendido como un conflicto de comunicación 
más que como un conflicto intra-individual, permite que se produzcan simultánea y relacionalmente conflictos lógicos y sociales. Así el conflicto sociocognitivo se considera como una vía privilegiada del desarrollo cognitivo; pero, no todas las situaciones que generan conflictos se resuelven en una elaboración de instrumentos cognitivos nuevos y más avanzados. Carugati y Mugny (1985) han especificado las condiciones idóneas en las que el progreso sociocognitivo queda asegurado:

a. heterogeneidad de los niveles cognitivos de los sujetos,

b. una oposición de centraciones,

c. la existencia de puntos de vista opuestos, o

d. cuestionar de forma sistemática el punto de vista del otro.

La efectividad de estas condiciones ha sido demostrada experimentalmente y tienen todas en común el que los sistemas de respuestas de los participantes en la interacción, sea real o simbólica, difieren (Mugny, De Paolis y Carugati, 1984). Hasta el punto de que «la inducción de un conflicto sociocognitivo es susceptible de hacer progresar al niño independientemente de la existencia de un problema cognitivo» (Carugati y Mugny, 1985; pág. 67). De este modo, las regulaciones cognitivas que se producen tras las interacciones responden a la necesidad de establecer o recomponer una relación social con el otro, de equilibrar una situación social específica.

«Nuestro estudio se encuadra, por una parte, dentro de este marco teórico, por lo que es copartícipe de su principal propósito: estudiar e investigar la "construcción social de las representaciones cognitivas"» (Mugny, 1985; pág. 15).

Por otra, es fruto de un proyecto ambicioso de investigación que se ha ido gestando desde 1984 y cuyo objetivo es el de trasladar los logros experimentales de susodicho programa, centrados primordialmente en las operaciones concretas [exceptuando, quizás, las experiencias de Cloutier y Goldschmid (1978) y Flieller (1986) sobre el esquema de proporciones], a la génesis del pensamiento adulto. En concreto, se ha plasmado en las investigaciones llevadas a cabo sobre el esquema de combinatoria (Fierro, Fernández y Santamaría, 1986) e inferencia causal (Fernández y Santamaría, 1986), y a las que esta investigación pretende completar y continuar, ciñéndose ahora al estudio del razonamiento inductivo, en concreto, al esquema de probabilidad.

\section{OBJETIVOS E HIPOTESIS}

El objetivo principal, pues, de este estudio reside en operacionalizar la función del conflicto sociocognitivo en la interacción social como factor causal en la génesis de la construcción del esquema formal de probabilidad.

Más específicamente, las hipótesis del estudio pueden agruparse en tres grupos:

\section{A. Referentes a la interacción social como factor causal en la construcción de los procesos cognitivos.}

Como hipótesis principales:

A.1. Las condiciones de interacción social facilitarán la adquisición del esquema de probabilidad más que las condiciones de trabajo individual (Doise y Mugny, 1981). 
A.2. Las condiciones de interacción social en las que el nivel cognitivo de los sujetos que interactúen sea heterogéneo facilitarán más la adquisición del esquema de probabilidad que cuando sea homogéneo (Carugati y Mugny, 1985).

A.3. Las condiciones de interacción social en las que la modalidad de su sistema relacional favorezca el conflicto de respuestas y obligue a sus componentes a resolverlo inducirá mayores logros en el esquema de probabilidad que cuando esto no ocurra (De Paolis y Mugny, 1985).

Como hipótesis secundarias:

A.4. La condición de interacción social en la que el nivel cognitivo de los sujetos que interactúen sea heterogéneo y la modalidad de su sistema relacional favorezca el conflicto de respuestas, obligando a sus componenetes a resolverlo, será la que mayores progresos provoque en el esquema de probabilidad (combinación de la hipótesis A.2 y A.3.).

A.5. Las condiciones de interacción en las que la modalidad de su sistema relacional no favorezca el conflicto de respuestas no se diferenciarán de las condiciones de trabajo individual en cuanto a los progresos inducidos en el esquema de probabilidad (Mugny, De Paolis y Carugati, 1984).

A.6. La complacencia, o regulación relacional de sumisión de un miembro de la interacción con respecto al otro, influirá negativamente en los logros cognitivos posteriores a la interacción del sujeto «sumiso» (Carugati, De paolis y Mugny, 1980-1981).

\section{B. Referentes a la superioridad de los logros obtenidos durante el trabajo grupal}

B.1. La productividad del trabajo en grupo con conflicto será de un nivel cognitivo superior a la del trabajo individual (Johnson et al., 1981; Slavin, 1983; Slavin et al., 1985).

\section{Referente a la generalización de los resultados.}

C.1. Los logros obtenidos por las diferentes situaciones experimentales en el esquema de probabilidad se generalizarán a los esquemas de proporciones, en especial, y al de covariación.

\section{METODO}

\section{Sujetos}

Los sujetos fueron 226 niños, de ambos sexos, alumnos del tercer ciclo de EGB en el Colegio Nacional «San José de Calasanz» de Málaga, colegio sito en una zona céntrica y al que acuden niños de clase media. La edad de los niños oscilaba de 11 a 15 años, siendo la media de edad de 12, 8 años.

A lo largo de las diferentes fases del experimento podrán apreciarse, por criterios de homogeneización de las muestras, primordialmente, así como por mortandad experimental, que la muestra quedó progresivamente reducida de tamaño, hasta llegar en la fase final a 200 sujetos.

\section{Procedimiento}

Todos los sujetos participaron en las tres fases del experimento: pretest, intervención y postest. 


\section{1}

\section{Primera fase: Pretest}

Los sujetos realizaban individualmente tres pruebas que versaban sobre las tareas de proporciones, probabilidad y covariación. La finalidad de esta fase era doble:

1. conocer el nivel cognitivo de los sujetos para poder asignarlos a grupos donde interaccionarán con susjetos de su mismo nivel cognitivo o diferente, según las condiciones experimentales, y

2. servir de medida de pre-tratamiento.

\section{Segunda fase: Intervención}

Se realizó 7 días después de la primera fase. A partir de la puntuación obtenida por los sujetos en el pretest, éstos fueron asignados al azar a seis condiciones experimentales:

Condición 1: Interacción social heterogénea con conflicto. En la que dos sujetos de nivel cognitivo diferente trabajan juntos en la resolución de una serie inacabable de problemas de probabilidad (con contenido distinto de las pruebas de pretest y postest, tal como se indica en la descripción de las pruebas); con la peculiaridad de que cada tres ítems se inducía una contradicción con las respuesta previas de los sujetos. Cada uno de los sujetos debía dar, en principio, una respuesta individual sobre la resolución del problema, para pasar a continuación a contrastarla con su compañero, discutir sobre su pertinencia y llegar a un acuerdo sobre la respuesta correcta.

Condición 2: Interacción social homogénea con conflicto. Idéntica a la anterior, salvo en que el nivel cognitivo de los componentes es el mismo.

Condición 3: Trabajo individual con conflicto. Los sujetos trabajan solos en la resolución de las tareas, aunque la estructura de la tarea les induce a un conflicto con sus respuestas previas cada tres ítems que deben solventar (se trata, pues, de un conflicto cognitivo).

Condición 4: Interacción social beterogénea sin conflicto. Los sujetos de nivel cognitivo diferente, como en la condición 1, trabajan en problemas en los que no se induce conflicto cada tres ítems, ni se les exige que se pongan de acuerdo en la toma de una respuesta conjunta.

Condición 5: Interacción social bomogénea sin conflicto. Estructura idéntica a la de la condición 4, aunque los sujetos son del mismo nivel cognitivo.

Condición 6: Trabajo individual sin conflicto. Los sujetos trabajan solos en la resolución de las tareas (como en la condición 3), aunque la estructura de la tarea no les induce a un conflicto cognitivo con sus respuestas previas.

En definitiva, se ha planteado como un diseño factorial incompleto $2 \times 2 \times 2$, dado que sólo se combinaron los factores formando seis condiciones experimentales de las ocho posibles. A los factores manipulados experimentalmente los denominaremos Interacción/Individual (si los sujetos trabajaban en grupo o solos), Conflicto/Sin conflicto (si la estructura de la tarea propiciaba situaciones de conflicto o no) y Heterogéneo/Homogéneo (si el nivel cognitivo de los sujetos que interaccionaban era diferente o similar). Por comodidad en la exposición de los resultados nos referiremos a ellos por el primero de sus valores, esto es, Interacción, Conflicto y Heterogéneo.

Todos los sujetos realizaban la tarea propuesta sin ninguna limitación de tiempo, aunque pasados 30 minutos se recogían las pruebas. 
Los sujetos que trabajaron por parejas realizaban la resolución de los problemas bajo la vigilancia y asesoramiento de un experimentador, el cual garantizaba que se cumplieran las características de cada condición. Este igualmente registraba las respuestas de los sujetos, así como el tipo de acuerdo al que habían llegado los sujetos cuando fuera pertinente (en la descripción de las pruebas se detalla la hoja de respuestas).

\section{Tercera fase: Postest.}

Se realizó 10 días después de la segunda fase y se pasaron unas pruebas paralelas a la del pretest para volver a medir los conceptos de proporciones, probabilidad y covariación.

\section{Descripción de las pruebas.}

Explicaremos, en primer lugar, las pruebas utilizadas para medir en el pretest y en el postest el nivel de los sujetos en proporciones, probabilidad y covariación; para, posteriormente, pasar a describir las utilizadas durante la segunda fase (o fase de intervención).

La tarea de proporciones que empleamos fue una adaptación de la tarea del Zumo de Naranjada de Noelting (1980a, b; Noelting, 1981) que consta de 12 ítems. Esta prueba ha sido utilizada y adaptada por diversos autores (Cloutier y Goldschmid, 1978; Corral, 1987; Fernández, 1990; Flieller, 1986; Pérez Echeverría, 1986; Pérez Echeverría, Carretero y Pozo, 1986), dada la fácil comprensión por los sujetos del procedimiento a seguir. El contenido de la prueba se modificó ligeramente, los sujetos en nuestra versión tenían que decidir entre dos naranjadas hechas al mezclar vasos de zumo de naranja y cucharadas de azúcar cuál era la más dulce o si estaban igual de dulces, mientras que en la de Noelting se mezclaban vasos de zumo de naranja y agua.

Para la tarea de probabilidad se utilizaron los mismos «cocientes» $(\mathrm{a} / \mathrm{b})$ que para los 12 ítems de la prueba de proporciones, pero con otra historia, con lo que se intentaba que la complejidad narrativa de las pruebas fuera casi idéntica. El contenido de la prueba versaba sobre averiguar en cuál de dos tarros, que contenían diferente número de bombones de trufa y de licor, era más probable coger bombones de licor o si era igual de probable.

La tarea de covariación que empleamos fue la de Shaklee y Hall (1983), que consta de 12 ítems de progresivo nivel de dificultad. La probabilidad de dar la respuesta correcta por puro azar era de .11 para los ítems más fáciles y de .005 para los más difíciles. El contenido de la prueba hacía referencia a la asociación de que diferentes plantas estuvieran sanas o enfermas según se les tratara con vitaminas o sin ellas.

En la segunda fase las pruebas utilizadas se basaban en el concepto de probabilidad y versaban sobre una gira musical de diferentes grupos de moda en el año 1987 por algunas ciudades españolas. La finalidad era averiguar de cada dos grupos que iban a actuar en una ciudad cuál sería más probable que fuera más aplaudido, conociendo la proporción de aplausos y de espectadores del año pasado en esa ciudad. Sobre esta tarea general se articularon dos pruebas:

1. Gira musical sin conflicto, en la que hay que predecir qué grupo musical de cada dos será más aplaudido este año en cada ciudad donde actúe. Por ejemplo, 
$\begin{array}{lll} & \text { ALASKA } & \text { MECANO } \\ \text { MALAGA } & 400 / 100 & 100 / 400\end{array}$

El numerador representa el número de espectadores que les aplaudieron y el denominador los que no lo hicieron en esta misma ciudad el año pasado.

2. Gira musical con conflicto, en la que hay que predecir qué grupo musical de cada dos será más aplaudido este año en cada ciudad donde actúe; pero, además se le exige al sujeto qué averigüe de los seis grupos que han actuado en esa ciudad el año pasado cual será el más aplaudido este año. Con ello se logra poner en contradicción sus respuestas previas para cada una de las comparaciones de dos grupos (e. g., Alaska/Mecano) con la total. Se trata, en todo caso, de un conflicto cognitivo leve. Por ejemplo, en la ciudad de Málaga actuaron el año pasado estos seis grupos musicales, se les pide a los sujetos que los compare, dos a dos, tal como se presentan;

\section{ALASKA/LOQUILLO \\ MECANO/HOMBRES-G OLEOLE/RAMONCIN}

Posteriormente se les pide que indique cual será más aplaudido de los seis. El conflicto se produce, pues, entre las respuestas parciales dadas a cada par de grupos musicales con la respuesta referida a la comparación de los seis grupos.

Las contestaciones a cada prueba se registraban en una hoja de respuestas, cuando se trataba de una de las condiciones de interacción el experimentador poseía una hoja de registro de respuestas en la que se recogían:

- la respuesta del sujeto $A$,

- la respuesta del sujeto $B$,

- la respuesta final a la que han llegado,

Cómo se había llegado a la resolución final; cabían tres posibilidades:

- que la hubiera impuesto $A$,

- que la hubiera impuesto B, o

- que hubiera sido por mutuo acuerdo.

La finalidad de registrar este tipo de conductas era la de saber como influía el mayor o menor grado de complacencia (léase, que la respuesta la hubiera impuesto uno de los participantes en la interacción) en los logros cognitivos subsiguientes a la interacción.

\section{RESULTADOS}

\section{Referentes a la superioridad de los logros obtenidos durante el trabajo grupal.}

Para comprobar si los resultados obtenidos por nuestra experiencia permitían o no confirmar nuestra hipótesis B.1., la productividad del trabajo grupal con conflicto sería superior a la del trabajo individual, realizamos un análisis de varianza mediante el método de regresión de la productividad incitada por las condiciones experimentales durante la fase de tratamiento. 


\section{4}

Dada las características del diseño (en cada condición experimental podrían encontrarse sujetos de un nivel cognitivo muy diferente) consideramos oportuno eliminar los efectos de las puntuaciones previas de los sujetos en tarea de probabilidad. Tal como aconsejan Cronbach y Furby (1970), Cole y Means (1981) y Flieller (1986), para este tipo de diseño es necesario emplear algún método de equiparación estadística, en concreto, las técnicas de covarianza. Nosotros hemos optado por el análisis de las puntuaciones residuales obtenidas mediante un análisis de regresión. Todos los análisis que requieran eliminar los efectos del nivel cognitivo previo de los sujetos se harán sobre las puntuaciones residuales, no obstante se indicará para cada análisis en el que se utilice.

El número de sujetos empleados en los análisis subsiguientes es de 200 , que son los que cumplimentaron las tres fases del experimento.

La Tabla 1 nos presenta las medias residuales de productividad durante la fase de tratamiento para cada una de las diferentes condiciones experimentales.

TABLA I

Diferencias globales de la productividad durante la fase de tratamiento entre las diferentes condiciones experimentales.

\begin{tabular}{lcc}
\hline Condiciones & Medias residuales & $\mathbf{N}$ \\
\hline Primera & .279 & 32 \\
\hline Segunda & .081 & 35 \\
\hline Tercera & -.004 & 32 \\
\hline Cuarta & -.123 & 31 \\
\hline Quinta & -.094 & 38 \\
\hline Sexta & -.133 & 32 \\
\hline & & 200 \\
\hline
\end{tabular}

El factor que más influyó en la productividad fue el Conflicto $[\mathrm{F}(1,196)=41.74, \mathrm{MSe}=.044, \mathrm{p}<.0001]$, seguido por Interacción $[\mathrm{F}(1,196)=10.4, \mathrm{MSe}=.044, \mathrm{p}<.001]$ y Heterogéneo $[\mathrm{F}(1,132)=5.18$, MSe $=.046 \mathrm{p}=.024]$. Se encontraron interacciones para la combinación de los factores Conflicto $\times$ Interacción $[\mathrm{F}(1,196)=5.81, \mathrm{MSe}=.044, \mathrm{p}=.017] \mathrm{y}$ Conflicto $\times$ Heterogéneo $[\mathrm{F}(1,132)=9.47, \mathrm{MSe}=.046, \mathrm{p}=.003]$.

El test a posteriori de Scheffé se utilizó para comparar las diferencias entre las medias de las condiciones. Para la interacción del factor Conflicto con Interacción y Heteroéneo encontramos que la condición 1 es significativamente la más productiva con respecto a las demás $(\mathrm{p}<.01)$ y la condición 2 con todas la condiciones sin conflicto ( $\mathrm{p}<.01$, condiciones 3,4 y 5 ). La condición 3 fue superior a la condición 4 y $6(\mathrm{p}<.01)$.

\section{Referentes a la interacción social como factor causal en la construcción de los procesos cognitivos}

Para analizar los efectos de las diferentes condiciones experimentales sobre el postest, y comprobar la hipótesis principal del estudio (hipótesis A.) se reali- 
zó un análisis de varianza de las puntuaciones residuales de la varible probabilidad.

En la Tabla 2 se presentan, de forma general, las medias residuales de la variable probabilidad para las diferentes condiciones experimentales.

TABLA II

Diferencias globales de los efectos de cada condición experimental en el postest (tarea de probabilidad).

\begin{tabular}{l|c}
\hline \multicolumn{1}{c|}{ Condiciones } & Medias residuales \\
\hline Primera & 1.149 \\
\hline Segunda & .432 \\
\hline Tercera & -.225 \\
\hline Cuarta & -.108 \\
\hline Quinta & -.488 \\
\hline Sexta & -.712 \\
\hline
\end{tabular}

La variable independiente Conflicto $[\mathrm{F}(1,132)=18.03, \mathrm{MSe}=2.22, \mathrm{p}<.0001]$ fue la que más incidió en los progresos en el postest para la variable probablilidad, seguida por la variable Interacción $[\mathrm{F}(1,196)=8.9, \mathrm{MSe}=2.37, \mathrm{p}=.003]$ y la variable Heterogéneo $[\mathrm{F}(1,132)=4.58, \mathrm{MSe}=2.22, \mathrm{p}=.034]$ No se encontraron interacciones.

En relación a las conductas registradas durante la interacción (sólo se analizaron las condiciones de interacción con conflicto) indicar que la complacencia se operacionalizó por el tanto por ciento que los sujetos acataban la decisión del otro sin discusión. El propósito de tal operacionalización era comprobar la hipótesis A.6 (la complacencia, o regulación relacional de sumisión de un miembro de la interacción con respecto al otro, influirá negativamente en los logros cognitivos posteriores a la interacción del sujeto «sumiso»), los resultados muestran que existe una correlación negativa $(-.30, \mathrm{p}<.05)$ con los resultados en el postest. De manera que a mayor complacencia que mostrara el sujeto menores serían los logros cognitivos subsiguientes a la interacción.

Los sujetos más predispuestos a aceptar sin discusión la respuesta del otro compañero se corresponden con los sujetos de un nivel cognitivo inferior en el esquema de probabilidad. Así en la condición 1, de niveles cognitivos heterogéneos, los sujetos de un nivel cognitivo superior en este esquema mostraron una media de 3.16 conductas de imposición de su criterio mientras que los de nivel inferior de 1.21 ( $\mathrm{p}<.001$ ). Para la condición 2 (en la que el nivel cognitivo de los sujetos era homogéneo) la media de respuestas de imposición fue de 1.73, muy similar a la de los sujetos de nivel cognitivo inferior de la condición 1.

\section{Referente a la generalización de los resultados}

La hipótesis C.1. (los logros obtenidos por las diferentes situaciones experimentales en el esquema de probabilidad se generalizarán a los esquemas de pro- 
porciones, en especial, y al de covariación) se comprobó en primer lugar con la tarea de proporciones.

Para ello se compararon las puntuaciones residuales de los sujetos en la tearea de proporciones tras trabajar en la tarea de entrenamiento en probabilidades, con la que hubieran sacado los sujetos en proporciones si no hubieran pasado por la fase de tratamiento. La Tabla 3 muestra las diferencias entre lo que los sujetos obtuvieron en proporciones y lo que hubieran sacado en esa tarea tras quitar los efectos de la fase de entrenamiento.

\section{TABLA III}

Diferencias de generalización para las condiciones experimentales del entrenamiento en probabilidades a la tarea de proporciones.

\begin{tabular}{l|c}
\hline \multicolumn{1}{c|}{ Condiciones } & T de contraste \\
\hline Primera & $5.705^{* *}$ \\
\hline Segunda & 1.244 \\
\hline Tercera & -.592 \\
\hline Cuarta & -.298 \\
\hline Quinta & $-1.766^{*}$ \\
\hline Sexta & $-2.759^{*}$ \\
\hline
\end{tabular}

$* \mathrm{p}<.05$

$* * \mathrm{p}<.00001$

La T positiva indica que la puntuación obtenida por los sujetos en la tarea de porporciones tras el entrenamiento en probabilidades es superior a la que los sujetos hubieran obtenido si no hubieran realizado el entrenamiento en esa condición experimental.

La T negativa, al contrario, supone que los sujetos habrían sacado mayor puntuación en proporciones si no hubieran realizado el entrenamiento correspondiente en probabilidades.

La generalización al esquema de proporciones inducida por la condición experimental de interacción social heterogénea con conflicto presenta una significación elevada $(\mathrm{p}<.00001)$, mientras que para la condición de interaccioón social homogénea con conflicto sólo nos encontramos con una tendencia de $\mathrm{p}=.11$.

Las condiciones 3 y 4 no presentan diferencias significativas, pero para las condiciones 5 y 6 se encuentran efectos negativos para el esquema de proporciones tras el entrenamiento en el esquema de probabilidad.

Con respecto a la tarea de covariación no se encontró ningún tipo de efectos de generalización de las condiciones de tratamiento en probabilidades.

\section{DISCUSION Y CONCLUSIONES}

\section{Referentes a la superioridad de los logros obtenidos durante el trabajo grupal}

La hipótesis B.1., la productividad del trabajo en grupo con conflicto será de un nivel cognitivo superior a la del trabajo individual, ha quedado confirma- 
da con un amplio margen de confianza. El análisis de la interacción de los factores Conflicto con Interacción social de mayor conflicto (la condición 1) es la que produjo mejores logros durante la interacción. En segundo lugar, la condición 2 que es la condición de interacción social que le sigue en grado de conflicto.

La interacción que hemos encontrado entre los factores manipulados experimentalmente señala claramente que no es suficiente con que las respuestas del compañero sean las adecuadas, requisito que se da en la condición 4 de interacción heterogénea sin conflicto, sino que es necesario una actividad de discusión, en definitiva, de conflicto sociocognitivo.

En este sentido, incluso a veces no es necesario el conflicto cognitivo. Como en la condición 2 de interacción social homogénea con conflicto, en la que el nivel cognitivo de los sujetos es el mismo, pero donde existe una dinámica social que les obliga a discutir sobre la tarea. Relación social de conflicto que repercute en logros cognitivos durante la interacción superiores a los que alcanzan ellos mismos individualmente.

Un resultado interesante de la experiencia realizada por nosotros es que la condición de trabajo individual con conflicto es superior a la condición 4 e igual a la condición 5 de interacción social sin conflicto. Por lo que habría que reformular la pregunta de Hill (1982) «Are $N+1$ heads better than one?» en ¿es mejor una «cabeza» que se cuestiona que $\mathrm{N}+1$ que no lo hacen?

En definitiva, la clave de la productividad grupal no está en el simple hecho de poner a trabajar a dos individuos «juntos», sino en especificar en qué «condiciones» se desarrollará ese trabajo.

\section{Referentes a la interacción social como factor causal en la construcción de los procesos cognitivos}

Las hipótesis del grupo A., que constituían las hipótesis principales del estudio, han salido ampliamente confirmadas. Con la peculiaridad de que en entrenamiento en probabilidades en las diferentes condiciones experimentales no se hizo con la misma tarea del pre y pos-tratamiento, sino con una tarea de contenido diferente.

Las condiciones de interacción con conflicto sociocognitio son las que mayores progresos cognitivos producen durante la interacción como hemos visto en los resultados de la fase segunda y son, a su vez, las que producen una mayor «interiorización» de esos logros interindividuales en intraindividuales.

El análisis realizado nos permite concluir que las situaciones de interacción social (hipótesis A.1.) en las que los componentes tienen niveles cognitivos diferentes (hipótesis A.2.) y presentan una modalidad de sistema relacional que favorece el conflicto de respuestas (hipótesis A.3.) son las que mayores logros cognitivos producen en el postest.

Pero no es necesario que el nivel cognitivo de los sujetos sea diferente para que se produzcan conflictos y subsiguientes progresos (Ames y Murray, 1982), tal como muestran los progresos alcanzados por los sujetos de la condición 2 de interacción social homogénea con conflicto, lo esencial es que el sistema de relaciones que existe en un grupo permita que las conductas de cada uno de los sujetos entre en conflicto con las del otro sin ningún tipo de supeditación o complacencia (tal como ha mostrado la correlación entre complacencia y logros cognitivos en el postest).

La condición de trabajo individual con conflicto cognitivo no es suficiente para lograr progresos significativos con respecto a los de la condición de trabajo 


\section{8}

individual sin conflicto cognitivo. Quizás hubiera sido necesario introducir un marcaje social en la tarea, como hizo en su expermento De Paolis (1982), para producir además de un posible conflicto cognitivo intraindividual un conflicto sociocognitivo.

Tras diez años de investigaciones volvemos a las conclusiones en las que incidían los primeros descubrimientos de Mugny y Doise (1978, 1979a): «no todas las interacciones sociales producen progresos». Este es el caso de las condiciones experimentales 4 y 5 de interacción social sin conflicto, hasta el punto de que son comparables a las condiciones de trabajo individual.

\section{Referente a la generalización de los resultados}

Hemos detectado unos progresos cognitivos en la tarea de probabilidad lo suficientemente relevantes como para que se generalicen a una tarea de proporciones. Dato especialmente interesante si tenemos en cuenta que el entrenamiento ha sido de 30 minutos y que los entrenamiento usuales en «proporciones», tal como señalan Tourniaire y Pulos (1985), son largos y la transfererencia de aprendizaje a otras tareas de proporciones escasa. Aunque no lo necesario como para producir progresos en la tarea de covariación.

\section{Implicaciones}

¿Cuáles son las condiciones de interacción que producen progresos cognitivos? Las que permiten la elaboración de conflictos sociocognitivos durante las interacciones, en nuestra experiencia las condiciones 1 y 2 . Esta afirmación tan tajante nos lleva inevitablemente a otra pregunta: ¿es el conflicto la única fuente de progresos cognitivos? Desde una perspectiva piagetiana (Piaget, 1975), el conflicto es la forma ideal de crear «perturbaciones» en el sistema de conocimiento, independientemente de si las interacciones del sujeto son con el medio físico o social. Para nosotros, fomentar la controversia durante las interacciones sociales es una de las estrategias más adecuadas para estimular en los contextos educativos la creación de conocimiento, mejorar las habilidades cognitivas y cambiar los puntos de vista de las personas. No obstante, y desde perspectivas más vygotskianas, no creemos que sea la única estrategia posible (Azmitia, 1988; Coll, 1984; Brown y Palincsar, 1989; Edwards y Mercer, 1987; Forman y Cazden, 1985; Tudge y Rogoff, 1989; Wertsch, 1985). Durante las interacciones sociales entre iguales el intercambio de ideas está regido por múltiples factores que propician la comunicación, la intersubjetividad y la construcción de conocimiento. Por lo tanto, resulta ingenuo pensar que en situaciones de aprendizaje tan imprevisibles como las de interacción social entre iguales el conflicto sociocognitivo sea el único catalizador de la interiorización del conocimiento.

Hemos respondido a varias preguntas, hemos obviado otras y, esperamos, haber suscitados algunas. En una investigación que tenemos en proyecto intentaremos dilucidar, mediante un enfoque microanalítico, los cursos de acción e interacción específicos que propician y generan la construcción de esquemas relacionales y cognitivos. 


\section{Referencias}

Ames, G. J. Y Murray, F. B. (1982). When two wrongs make a right: promoting cognitive change by social conflict. Developmental Psychology, 18, 894-897.

Anderson. J. R. (1980). Cognitive Psychology and its implications. San Francisco: Freeman.

Azmitia, M. (1988). Peer interaction and problem solving: when are two heads better than one? Child Development, 59, 87-96.

BALDwIN, J. M. (1913). History of psychology. London: Watts.

BERLYNE, D. (1957). Uncertainty and conflict: A point of contact between information theory and behavior - theory concepts. Psychological Review, 64, 329-33.

Brown, A.L. Y Palincsar, A. S. (1989). Guided, coopertive learning and individual knowledge acquisition. En L. B. Resnick (Ed.), Knowing, learning and instruction. London: LEA.

Carugati, F. Y Mugny, G. (1985). La théorie du conflit sociocognitif. En G. Mugny (Ed.) Psychologie sociale du développement cognitif. Berne: Peter Lang.

Carugati, F., De Paolis, P. Y Mugny, G. (1980-1981). Conflit de centrations et relationnelles, III: Régulations cognitives et relationnelles du conflit socio-cognitif. Bulletin de Psychologie, 352, 843.85.

Cattaneo, C. (1864). Dell' antitesi come metodo di psicología sociale. Il Politecnico, 20, 262-27.

Cloutier, R. Y Goldschmid, M. (1978). Training proportionality through peer interaction. Instructional Science, 7, 127-142.

Cole, M. Y Means, B. (1981). Comparative studies of how people think. Harvard: Harvard University Press (Trad. cast. en Paidós, 1986).

Coll, C. (1984). Estructura grupal, interacción entre alumnos y aprendizaje escolar. Infancia y Aprendizaje, 27-28, 119-138.

CORral, A. (1987). El aprendizaje y la estrategia de comparación de proporciones. Infancia y Aprendizaje, 37. 33-43.

Cronbach, L. J. Y Furby, L. (1970). How we should measure «change», or should we? Psychological Bulletin, 74, 50-68.

De PAOLIS, P. (1982). Marquaje social et développement cognitif: Ler conflit sociocognitif induit par la représentation de normes socialles. Geneve Colloque Nouvelles Perspectives dans l'étude expérimentale du développment social de l'intelligence.

De Paolis, P. Y Mugny, G. (1985). Régulations relationnelles et sociocognitives du conflit cognitif, et marquaje social. En G. Mugny (Ed.), Psychologie sociale du développemnent cog. nitif. Berne: Peter Lang.

DE VEGA, M. (1985). Procesamiento de la información y cultura: Hacia una nueva integra. ción teórica. En J. Mayor (Ed.), Actividad bumana y procesos cognitivos. (Homenaje a J. L. Pinillos). Madrid: Alhambra Universidad.

Doise, W. Y Mugny, G. (1981). Le développment social de l'intelligence. Paris: Interéditions. (Trad. cast. en Trillas, 1983).

ECHEITA, G. (1988). El mundo adulto en la mente de los niños. La comprensión infantil de las relaciones de intercambio. Madrid: MEC-CIDE.

Edwards, C. Y Mercer, N. (1987). Common knowledge. London: Methuen. (Trad. cast. en Paidós, 1988).

FERNÁNDEZ, P. (1990). Relaciones teórico-empíricas entre los esquemas de proporción, probabilidad y covariación. Revista de Psicología Geneal y Aplicada, 43, 331-37.

Fernández, P. Y Santamaría, C. (1986). El pensamiento adulto en interacción: Inferencia causal. Resumen publicado en II Jornadas Internacionales de Psicología y Educación. Madrid.

Fierro, A., Fernández, P. Y Santamaría, C. (1986). Efectos de una experiencia interactiva en la adquisición de reglas formales de combinatoria. Revista de Psicología General y Aplicada, 41, 871-883.

Flieller, A. (1986). 'La coeducation de l'intelligence. Nancy: Presses Univer. de Nacy.

Fodor, J. A. (1983). The modularity of mind. Cambridge: M.I.T. Press. (Trad. cast. en Morata, 1986).

Forman, E. A. Y Garden, C. B. (1985). Exploring Vygotskian perspectives in education: The cognitive value of peer interaction. En J. V. Wertsch (Ed.), Culture, communication and cognition: Vygotskian perspectives. New York: Cambridge University Press.

HiLl, G. W. (1982). Group versus individual performance: Are N + 1 heads better than one? Psychological Buletin, 91, 517-53.

Johnson, D. W.; Maruyama, G.; Johnson, R. Y Nelson, D.(1981). Effects of cooperative, competitive, and individualistic goal structures on achievement: A meta-analysis. Psychological Bulletin, 89, 47-62.

Kunn, D. (1972). Mechanisms of change in the development of cognitive structures. Cbild Development, 43, 833-84. 


\section{0}

Lave, J. (1988). Cognition in practice. Cambridge: Cambridge University Press.

Mayor, J. (Ed.) (1985). Actividad bumana y procesos cognitivos. (Homenaje a J. L. Pinillos). Madrid: Alhambra Universidad.

Mugny, G. (Coord.) (1985). Psychologie sociale du développement cognitif. Berne: Peter Lang.

Mugny, G. Y Doise, W. (1978a). Socio-cognitive conflict and structure of individual and collective performances. European Journal of Social Psychology, 8, 181-19.

MugnY, G. Y DoIsE, W. (1979a). Factores sociológicos y psicosociológicos en el desarrollo cognitivo: Una nueva ilustración experimental. Anuario de Psicología, 21, 5-25.

Mugny, G., De Paolis, P. Y Carugati, F. (1984). Social regulations in cognitive development. En W. Doise y A. Palmonari (Eds.), Social interaction in individual development. Cambridge: Cambridge University Press.

Murray, F. B. (1972). Acquisiton of conservation through social interaction. Developmental Psychology, 6, 1-6.

Noelting, G. (1980a). The development of proportional reasoning and the ratio concept. Parte I: Differentiation of stages. Educationa Studies in Matbematics, 11, 217-25.

Noelting, G. (1980b). The development of proportional reasoning and the ratio concept. Parte II: Problem-structure at successive stages. Problem-solving strategies and the mechanism of adaptive restructuring. Educational Studies in Mathematics, 11, 331-36.

Noelting, G. (1981). Qualitative and quantitative aspects inb the development of proportional reasoning. En M. P. Friedman, J. P. Dasy N. O'Connor (Eds.), Intelligence and learning. New York: Plenum Press.

Piaget, J. (1923). El lenguaje y el pensamiento en el niño. Buenos Aires: Guadalupe, 1976.

PlaGeT, J. (1924). El juicio y el razonamiento en el niño. Buenos Aires: Guadalupe, 1972.

PlageT, J. (1932). el criterio moral en el niño. Barcelona: Fontanella, 1977.

Piaget, J. (1975). La equilibración de las estructuras cognitivas. Problema central del desarrollo. Madrid: Siglo XXI, 1978.

Perez Echevarria, M. P. (1986), Relación entre estrategias de cálculo proporcional y tipo de problema. Resumen publicado en II Jornadas Internacionales de Psicología y Educación. Madrid.

Pérez Echevarria, M. P., Carretero, M. Y Pozo, J. I. (1986). Los adolescentes ante las matemáticas: Proporción y probabilidad. Cuadernos de Pedagogía, 133, 9-13.

Perret.Clermont, A. N. (1979). La construcción de l'intelligence dans l'interaction sociale. Berne: Peter Lang. (Trad. cast. en Aprendizaje Visor, 1984.)

Riviere, A. (1987). El sujeto de la psicología cognitiva. Madrid: Alianza Psicología.

Seodne, J. (1985). Conocimiento y representación social,. En J. Mayor (Ed.), Actividad bumana y procesos cognitivos. (Homenaje a J. L. Pinillos). Madrid: Alhambra Universidad.

SHAKLEE, H. Y HALl, L. (1983). Methods of assessing strategies for judging covariation bet ween events. Journal of Educational Psychology, 75, 583-59.

Slavin, R. E. (1983). Cooperative learning. New York: Longivan.

Slavin, R.; Sharan, S.; Kagan, S. Y Cols. (1985). Learning to cooperate, cooperating to learn. New York: Plenum Press.

Smith, K.; Johnson, D. W. Y Johnson, R. T. (1981). Can conflict be constructive? Contro versy versus concurrence seeking in learning groups. Journal of Educational Psychology, 73, 651-66.

Toruniaire. F. Y Pulos, S. (1985). Proportional reasoning: A review of the literature. Educational Studies in Mathematics, 16, 181-20.

Tudge, J. Y Rogoff, B. (1989). Peer influences on cognitive development: piagetian and vygotskian perspectives. En M. H. Bornstein y J. Bruner (Eds.), Interaction in buman development. London: LEA.

Vygotski, L. S. (1934). Thought and language. Cambridge: M.I.T. Press. (Trad. cast. en Pléyade, 1973);

Vygotski, L. S. (1978). Mind in society: The development of bigher psychological processes. Harvard: Harvard University Press. (Trad. cast. en Crítica, 1979).

WaLLON, H. (1947). L'étude psychologique et sociologique de l'enfant. Cabiers Internationaux de Sociologie, 3, 3-23.

WERTSCH, J. V. (1979). From social interaction to higher psychological processes. A clarification and application of Vygotsky's theory. Human Development, 22, 1-22.

WerTsCH, J. V. (1985). Introducción. En J. V. Wertsch, Culture, communication and cognition: Vygotskian perspectives. New York: Cambridge University Press. 